\title{
Prevalence and family factors associated with loneliness during COVID-19: A cross-sectional study from South India
}

\author{
G Sanjana $^{1}$, Vijaya Raghavan ${ }^{1}$
}

\begin{abstract}
Background: Loneliness can affect anyone at any point in their life. It can be detrimental to the wellbeing and quality of life of individuals and communities. In the ongoing COVID-19 pandemic, loneliness is considered as a public health crisis. Hence, the objectives of the study were to estimate the prevalence of loneliness and family related factors associated with loneliness among general population in south India.
\end{abstract}

Materials and Methods: The study employed a crosssectional online survey design. The data was collected in the first phase of the lockdown in 2020 from adults in Southern India. Socio-demographic profile and family related variables were collected using a semistructured proforma. Loneliness was assessed by UCLA loneliness scale.

Results: Of 573 total respondents to the survey, aged between $18-65$ years, $43 \%$ were male and $57 \%$ were female. The overall prevalence of loneliness was $63 \%$ (358/573). No significant gender differences were observed in the prevalence of loneliness. Family discord was associated with higher rates of loneliness $(p<0.01)$. Other factors associated were younger age and being single.

Conclusion: Rates of loneliness during the COVID-19 lockdown were high in Southern India. Findings suggest that interventions should prioritize younger people. Increasing social support and improving interpersonal skills, which in turn would help reduce family discord and may reduce the impact of COVID-19 on loneliness.

Keywords: COVID-19, loneliness, family, isolation, lock-down, social, India
Running title: Loneliness and family factors during COVID-19

Address for Correspondence: Dr. G Sanjana, Schizophrenia Research Foundation, R/7A, North Main Road, Anna Nagar West Extension, Chennai - 600101, Tamil Nadu, India; E mail: gsanjana@ scarfindia.org

How to Cite this article: Sanjana G and Vijaya Raghavan. Prevalence and family factors associated with loneliness during COVID-19: A cross-sectional study from South India. Indian Journal of Mental Health and Neurosciences. 2020;3(2): pp 61-67

\section{INTRODUCTION}

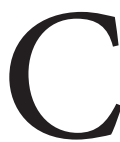

OVID-19 is caused by a novel coronavirus, named severe acute respiratory syndrome coronavirus 2 (SARS-CoV-2). ${ }^{1}$ At present, COVID-19 has led to a large number of deaths with tens of thousands of confirmed cases globally, giving rise to a serious public health crisis. The control of COVID-19 largely relies upon avoiding or minimizing physical contact with people. ${ }^{2}$ Therefore, a nationwide lockdown was imposed in India to contain the spread of the virus from March $25^{\text {th }}$ to May $31^{\text {st }}, 2020 .^{3}$ The social isolation, along with the financial and psychosocial burden, flared up after the COVI-19 lockdown, which was further compounded by the rising number of cases and mass panic and anxiety in the country. ${ }^{4}$

Loneliness is defined as an unpleasant experience that derives from deficiencies (qualitative and quantitative) in one's network of social relationships. ${ }^{5}$ Social integration and connections are imperative for an individual's development and well-being across the lifespan. Research suggests that loneliness can adversely

\footnotetext{
${ }^{1}$ Schizophrenia Research Foundation, Anna Nagar West Extension, Chennai, Tamilnadu.
} 
influence an individual's physical, mental and cognitive health. ${ }^{6}$ Loneliness has been associated with a greater risk of cardiovascular disease, dementia, anxiety and depression. ${ }^{7}$ Loneliness can impair daily activities and functioning and even exacerbate already existing mental health conditions. ${ }^{7}$

With restricted interpersonal contact opportunities and physical distancing in place, rates of loneliness increased all around the world during the COVID-19 pandemic. Recent evidence reported high levels of loneliness and stress in the UK. ${ }^{8} \mathrm{~A}$ study in Spain found that perception of self as a burden, lower quality of sleep and being more exposed to news about COVID-19 were associated with higher feelings of loneliness. ${ }^{9}$ In a survey among adolescents in Belgium during the COVID-19 quarantine found that participants who experienced loneliness were more inclined to use social media to cope with lack of social contact. ${ }^{10} \mathrm{~A}$ study among adults in Italy during the lockdown period, participants with perceived loneliness reported spending more time on social media which was also associated with increasing levels of anxiety. ${ }^{11}$

Very few studies have explored loneliness among general population during the ongoing COVID-19 pandemic in India. ${ }^{12,13}$ Understanding the magnitude of the problem at hand and the factors associated with loneliness will help in the development of interventions to address loneliness. Hence, the aim of the study was to estimate the prevalence of loneliness among general population and to examine the family related factors associated with loneliness in south India during the COVID-19 lockdown.

\section{MATERIALS AND METHODS}

Survey: An online survey was created and circulated from May 5 to June 15, 2020. The survey questions were circulated using google forms.

Inclusion and Exclusion Criteria: Subjects with the following inclusion criteria were included in the study:
1) Age 18 to 65 years; 2) Residing in South India during the COVID-19 lockdown; 3) Any gender; and 4) consent to be a part of the study.

Measures: the following data was recorded

Socio-demographic and family variables: The online survey included information about socio-demographic profile and family related factors such as 'How much has COVID-19 affected your family in terms of stress and discord? 'Does your home environment feel tense?' 'Are you finding relationships with close ones difficult?' and 'In your relationship - how much are you able to express your needs (both physical and emotional)?'

Loneliness: Loneliness was assessed by UCLA Loneliness Scale. ${ }^{14}$ It is a 20 -item scale used globally to measure one's subjective feelings of loneliness and social isolation. The scale uses a 4 -point rating scale ( $1=$ never; $4=$ always).

Statistical Analysis: All the statistical analyses were done using SPSS 20.0. Mean and standard deviation were used to summarize continuous variables and frequency and percentage were used to summarize categorical variables. Chi-square test was used to find association between categorical variables and Pearson's correlation for continuous variables. A $p<0.05$ was set as statistically significant.

\section{RESULT}

\section{Socio demographic profile and family related variables}

A total of 573 respondents took part in the study. The socio-demographic profile of the respondents is depicted in Table 1. Almost equal proportions of participants were employed (50.6\%) and unemployed (49.4\%). The mean age of the respondents was 28 years. Two-thirds (67.5\%) of the participants in the age group $18-30$ and $42.5 \%$ of the participants above the age 30 reported feeling lonely during the lockdown. Among the respondents, 322 were single and 251 were in a relationship. Among the respondents, $2.4 \%$ lived alone. 
Table 1. Socio-demographic profile of the study participants $(N=573)$

\begin{tabular}{|l|c|}
\hline \multicolumn{1}{|c|}{ Variables } & Frequency (\%); Mean \pm SD \\
\hline Gender, N (\%) & $246(42.8)$ \\
\hline Male & $326(56.7)$ \\
\hline Female & $1(0.5)$ \\
\hline Others & $27.92 \pm 9.87$ \\
\hline Age (in years) & $311(54.3)$ \\
\hline Current relationship status & $14(2.4)$ \\
\hline Single & $73(12.7)$ \\
\hline \multirow{2}{*}{ In a relationship } & $164(28.6)$ \\
\hline Married & $7(1.2)$ \\
\hline Separated/ Divorced & $4(0.7)$ \\
\hline Widowed & \\
\hline Current living status & $14(2.4)$ \\
\hline Alone & $280(48.9)$ \\
\hline Less than or equal to 4 & $279(48.7)$ \\
\hline More than 4 & \\
\hline Current employment status & $290(50.7)$ \\
\hline Employed & $31(5.4)$ \\
\hline Unemployed & $45(7.8)$ \\
\hline Homemaker & \\
\hline
\end{tabular}

Prevalence of loneliness

The overall prevalence of loneliness in the population was $62.5 \%$. The prevalence of loneliness was higher among respondents between the ages 18-30 as compared to participants above age 30 (Table 2). Respondents who were single were found have significantly higher prevalence of loneliness when compared with respondents who were in a relationship. No gender differences were observed in the prevalence of loneliness.

Table 2. Prevalence of Loneliness and its association between socio-demographic profile among the study participants $(\mathrm{N}=573)$

\begin{tabular}{|c|c|c|c|c|c|}
\hline Variables & $\begin{array}{l}\text { Total } \\
\text { n (\%) }\end{array}$ & $\begin{array}{c}\text { No Loneliness } \\
\text { n (\%) }\end{array}$ & $\begin{array}{c}\text { Loneliness } \\
\text { n (\%) }\end{array}$ & \multirow[t]{2}{*}{$p$ value } & \multirow[t]{2}{*}{ CI } \\
\hline $\mathrm{N}(\%)$ & $573(100)$ & $215(37.5)$ & $358(62.5)$ & & \\
\hline \multicolumn{6}{|l|}{ Gender } \\
\hline Male & 246 & $87(35.4)$ & $159(64.6)$ & \multirow{2}{*}{0.341} & $0.601-$ \\
\hline Female & 326 & $128(39.3)$ & $198(60.7)$ & & 1.193 \\
\hline \multicolumn{6}{|l|}{ Age } \\
\hline $18-30$ & 421 & $137(32.5)$ & $284(67.5)$ & \multirow{2}{*}{0.001} & $0.314-$ \\
\hline $30-65$ & 152 & $78(51.3)$ & $74(48.7)$ & & 0.668 \\
\hline \multicolumn{6}{|c|}{ Relationship status } \\
\hline Single & 322 & $98(30.4)$ & $224(69.6)$ & \multirow[b]{2}{*}{$<0.001$} & \multirow{2}{*}{$\begin{array}{r}0.355- \\
0.706\end{array}$} \\
\hline $\begin{array}{ll}\text { In } & a \\
\text { relationship }\end{array}$ & 251 & $117(46.6)$ & $134(53.4)$ & & \\
\hline
\end{tabular}




\section{Association between family related factors and loneliness}

Only $29.6 \%$ of the population that reported feeling lonely faced no family stress and discord. $59 \%$ of the population faced mild, $8.7 \%$ faced moderate family discord, with outbursts being more frequent and children getting in physical fights. $2.8 \%$ of the population participants faced severe familial discord in which hitting and inducing harm were reported. Table 3 depicts other family factors associated with loneliness. Finding relationships with close ones difficult, feeling dependent on others, a loss of control/power, not being able to openly speak about ones' problems were all associated with feelings of loneliness. $42 \%$ of the population that reported feeling lonely found their environment tense. The study also explored emotional and physical expressing of needs among participants who were in a relationship; it was found that $5.3 \%$ were not able to express at all, $9.5 \%$ to a small extent, $24.6 \%$ to some extent and $11.2 \%$ were able to express to a great extent.

\section{Table 3. Association between family variables and loneliness among the study participants}

\begin{tabular}{|c|c|c|c|c|}
\hline Variables & No Loneliness & Loneliness & p value & 95\% CI \\
\hline \multicolumn{5}{|c|}{ Does your environment feel tense? } \\
\hline Yes & $54(25.1)$ & $149(41.6)$ & \multirow{2}{*}{0.002} & \multirow{2}{*}{$0.324-0.683$} \\
\hline No & $161(74.9)$ & 209(58.4) & & \\
\hline \multicolumn{5}{|c|}{ Are you finding relationships with close ones difficult? } \\
\hline Yes & $20(9.3)$ & $132(36.9)$ & \multirow{2}{*}{$<0.001$} & \multirow{2}{*}{$0.106-0.292$} \\
\hline No & 195(90.7) & $226(63.1)$ & & \\
\hline \multicolumn{5}{|c|}{ I feel dependent on others } \\
\hline Yes & $62(28.8)$ & $149(41.6)$ & \multirow{2}{*}{0.002} & \multirow{2}{*}{$0.396-0.817$} \\
\hline No & $153(71.2)$ & $209(58.4)$ & & \\
\hline \multicolumn{5}{|c|}{ I feel loss of control/power } \\
\hline Yes & $41(19.1)$ & $137(38.3)$ & \multirow{2}{*}{$<0.001$} & \multirow{2}{*}{$0.254-0.568$} \\
\hline No & $174(80.9)$ & $221(61.7)$ & & \\
\hline \multicolumn{5}{|c|}{ I am speaking openly about my problems } \\
\hline Yes & $151(70.2)$ & $157(43.9)$ & \multirow{2}{*}{$<0.001$} & \multirow{2}{*}{$2.109-4.326$} \\
\hline No & $64(29.8)$ & $201(56.1)$ & & \\
\hline \multicolumn{5}{|c|}{ How much has COVID-19 affected your family in terms of stress and discord } \\
\hline No & $115(53.5)$ & $106(29.6)$ & \multirow{4}{*}{$<0.001$} & \multirow{4}{*}{ - } \\
\hline Mild & $89(41.4)$ & 211(58.9) & & \\
\hline Moderate & $9(4.2)$ & $31(8.7)$ & & \\
\hline Severe & $2(0.9)$ & $10(2.8)$ & & \\
\hline \multicolumn{5}{|c|}{ In your relationship - how much are you able to express your needs (both physical and emotional? } \\
\hline Not at all & $5(2.3 \%)$ & $19(5.3)$ & \multirow{5}{*}{$<0.001$} & \multirow{5}{*}{ - } \\
\hline Small extent & $7(3.3)$ & $34(9.5)$ & & \\
\hline Some & $29(13.5 \%)$ & $88(24.6)$ & & \\
\hline Great & $88(40.9 \%)$ & $40(11.2 \%)$ & & \\
\hline N/A & $86(40.0 \%)$ & $177(49.4)$ & & \\
\hline
\end{tabular}




\section{DISCUSSION}

The aim of the study was to estimate the prevalence of loneliness and its association with family related factors amongst adults aged 18-65 during the COVID-19 lockdown in Southern India.

Results show that the overall prevalence of loneliness was $62.5 \%$ during the COVID-19 lockdown in South India. In another study conducted in the earlier phase of the lockdown in Japan, it was found that over $40 \%$ (41.4\%) of the Japanese adults were lonely. ${ }^{15}$ In a comparative study from Hong Kong, the prevalence of loneliness pre-COVID-19 phase was $40.6 \%$ and peri-COVID-19 phase was $71.1 \%$ among older adults. ${ }^{16}$ Recent research published in the United Kingdom show a prevalence rate of $27 \%$ for loneliness during the lockdown. ${ }^{8}$

The high prevalence of loneliness in this study which is comparable with the other Asian countries could be due to the fact that they are collectivistic nations emphasizing on cultural and social ties. ${ }^{17,}{ }^{18}$ However, with urbanization and migration, and social structure rampantly changing, the risk of loneliness increases. ${ }^{19}$ The lower prevalence of loneliness in the UK when compared to India could be due to cross-cultural differences that emphasize on individualistic societies. ${ }^{20}$

Results show that as the severity of family discord increases, the levels of loneliness increase. Similar results were observed in Taiwan, which showed that that those with stronger family cohesion during early adolescence were less likely to suffer from serious loneliness in young adulthood. ${ }^{21}$

With familial discord and an unhealthy family climate, family members may perceive a discrepancy in the quality of interactions that they have with one another, which may lead them to self-identify as lonely. The uncertainty of the pandemic, financial and academic stress, lack of personal space, resurfacing of past trauma, differences in ideologies and values among family members may have influenced the family climate leading to loneliness. ${ }^{22}$

In the study, no gender differences in loneliness experienced, younger age group $(<30$ years of age) and being single were found to be associated with higher prevalence of loneliness. Studies in Spain and UK have found that women reported higher feelings of loneliness; however, no differential impact on gender was observed in our study. This may be due to the major cultural influences and norms in India. Women may not acknowledge and express feelings of loneliness due to the stigma and socio-cultural taboo surrounding it. It may also reflect the difficulty in voicing one's own concern and feeling burdensome to the family members.

Consistent with previous studies, those who had a spouse, or a partner were found to show lower levels of loneliness as opposed to those who were single. This reflects that being in a relationship can act as a protective factor. The companionship and intimacy of a partner reduces the feelings of loneliness. Considering the study was done with individuals who had access to the internet and social media, it indicates that the digital connectivity does not necessarily translate to a reduction in perceived loneliness.

An inverse relationship with age and loneliness was found, with very high prevalence of loneliness among the younger age group. The prevalence of loneliness decreased with age with 18-30-year old's having the highest frequency of loneliness (67.5\%). Younger adults may be affected disproportionately due to the cessation of opportunities to visit schools, colleges or workplaces where they could interact and socialize. It is also important to consider that as young adults one's social needs are much higher and emotional regulation is not fully developed. ${ }^{23}$

Studies from the UK, Spain along with our current study have found that the youth is at higher risk for loneliness. ${ }^{8}$, ${ }^{9}$ This is also an indicator that the interventions to alleviate loneliness should target younger people. A good starting point is by addressing loneliness and isolation in the classrooms and workplaces.

Limitations of the current study are: 1 . This was a crosssectional study done with individuals who have access to the internet. Hence, the results will be not generalisable to all sections of population; and 2. The age range of the participants included is restricted and not included children.

More follow-up studies can be done to assess the risk factors and impact of loneliness. This study will also help us in setting out immediate priorities and longterm strategies for social connectedness and well-being at the community level.

\section{CONCLUSION}

More than two-thirds of the population reported feeling lonely in Southern India. Family discord has also been 
found to be associated with loneliness. This can be detrimental to physical and mental health as well as how communities function at large. Therefore, novel interventions using digital platforms that provide a sense of belongingness and enhance community engagement should be developed. It is essential to place loneliness and social relationships high on the wider public mental health and research agenda.

\section{Acknowledgement: None}

Source of funding: This research has not received specific financial grant from any funding agency in the public, commercial or not-for-profit sectors

Conflict of interest: The authors have declared no conflict of interest with respect to the research, authorship, and/or publication of this article.

\section{REFERENCES}

1 Lai C-C, Shih T-P, Ko W-C, et al. Severe acute respiratory syndrome coronavirus 2 (SARS-CoV-2) and corona virus disease-2019 (COVID-19): the epidemic and the challenges. International Journal of Antimicrobial Agents. 2020;55(3):105924

2 Chu DK, Akl EA, Duda S, et al. Physical distancing, face masks, and eye protection to prevent person-toperson transmission of SARS-CoV-2 and COVID-19: a systematic review and meta-analysis. The Lancet. 2020;395(10242):1973-1987

3 Ghosh P, Ghosh R, and Chakraborty B. COVID-19 in India: State-wise Analysis and Prediction. JMIR Public Health and Surveillance. 2020;6(3):e20341

4 Dubey S, Biswas P, Ghosh R, et al. Psychosocial impact of COVID-19. Diabetes and Metabolic Syndrome. 2020;14(5):779-788

5 Peplau LA and Perlman D. Theoretical approaches to loneliness. Loneliness: A sourcebook of current theory, research and therapy. New York, Wiley. 1982:123-34

6 Hawkley LC, Cacioppo JT. Loneliness matters: A theoretical and empirical review of consequences and mechanisms. Annals of Behavioral Medicine. 2010;40(2):218-227

7 Mushtaq R, Shoib S, Shah T, et al. Relationship between loneliness, psychiatric disorders and physical health? A review on the psychological aspects of loneliness. Journal of Clinical Diagnosis and Research. 2014;8(9):WE01-WE04

8 Groarke JM, Berry E, Graham-Wisener L, et al. Loneliness in the UK during the COVID-19 pandemic: Cross-sectional results from the COVID-19 Psychological Wellbeing Study. PLoS One. 2020;15(9):e0239698

9 Losada-Baltar A, Jiménez-Gonzalo L, Gallego-Alberto L, Pet al. “We’re staying at home”. Association of selfperceptions of aging, personal and family resources and loneliness with psychological distress during the lock-down period of COVID-19. The Journals of Gerontology: Series B, Psychological Sciences and Social Sciences. [epub ahead of print].

10 Cauberghe V, Van Wesenbeeck I, De Jans S, et al. How adolescents use social media to cope with feelings of loneliness and anxiety during COVID-19 lockdown. Cyberpsychology, Behavior and Social Networking. [epub ahead of print]. doi: 10.1089/cyber.2020.0478.

11 Boursier V, Gioia F, Musetti A, et al. Facing loneliness and anxiety during the COVID-19 isolation: the role of excessive social media use in a sample of Italian adults. Frontiers in Psychiatry. 2020;11.586222

12 Banerjee D, Rai M. Social isolation in Covid-19: The impact of loneliness. International Journal of Social Psychiatry. 2020;66(6):525-527

13 Pandey D, Bansal S, Goyal S, et al. Psychological impact of mass quarantine on population during pandemicsThe COVID-19 Lock-Down (COLD) study. PLoS One. 2020;15(10):e0240501

14 Russell DW. UCLA Loneliness Scale (Version 3): Reliability, validity, and factor structure. Journal of Personality Assessment. 1996;66(1):20-40 
15 Stickley A, Matsubayashi T, Ueda M. Loneliness and COVID-19 preventive behaviours among Japanese adults. Journal of Public Health (Oxford, England). 2020;43(1):53-60

16 Wong SYS, Zhang D, Sit RWS, et al. Impact of COVID-19 on loneliness, mental health, and health service utilisation: a prospective cohort study of older adults with multimorbidity in primary care. British Journal of General Practice. 2020;70(700):e817-e24

17 Lykes VA, Kemmelmeier M. What predicts loneliness? Cultural difference between individualistic and collectivistic societies in Europe. Journal of Cross-Cultural Psychology. 2014;45(3):468-490

18 Barreto M, Victor C, Hammond C, et al. Loneliness around the world: Age, gender, and cultural differences in loneliness. Personality and Individual Differences. [epub ahead of print]. doi: 10.1016/j.paid.2020.110066

19 Zhong B, Xu Y, Jin D, et al. Prevalence and correlates of loneliness among Chinese service industry migrant workers: A cross-sectional survey. Medicine. 2016;95(24).e3903

20 Heu LC, van Zomeren M, Hansen N. Lonely alone or lonely together? A Cultural-psychological examination of individualism-Collectivism and loneliness in five European countries. Personality and Social Psychology Bulletin. 2019;45(5):780-793

21 Chiao C, Chen Y-H, Yi C-C. Loneliness in young adulthood: Its intersecting forms and its association with psychological well-being and family characteristics in Northern Taiwan. PLoS One. 2019;14(5):e0217777

22 Sarner M. Maintaining mental health in the time of coronavirus. New Scientist. 2020;246(3279):40-46

23 Theurel A and Gentaz E. The regulation of emotions in adolescents: Age differences and emotion-specific patterns. PLoS One. 2018;13(6):e0195501 